\title{
Esophageal Varices: retrospective analysis of cases in Damascus Hospital and a review of the literature.
}

\section{Assem Kubtan}

Departement of surgery, Faculty of medicine, Syrian Private University, Damascus, Syria

Fahd dbiss ( $\square$ Fahddbess@gmail.com )

Faculty of medicine, Syrian Private University, Damascus, Syria

\section{Research Article}

Keywords: Syria, esophageal varices, surgery

Posted Date: February 2nd, 2022

DOI: https://doi.org/10.21203/rs.3.rs-1302626/v1

License: (1) This work is licensed under a Creative Commons Attribution 4.0 International License. Read Full License 


\section{Abstract \\ Background:}

Esophageal varices are abnormal, enlarged veins in the tube that connects the throat and stomach (esophagus). This condition occurs most often in people with serious liver diseases. Hepatitis, alcohol, and obesity are well documented risk factors for esophageal varices. Endoscopic variceal ligation (EVL) monotherapy is the standard therapy for managing esophageal variceal hemorrhage. This study aims to evaluate esophageal varices risk factors and the used methods in treating esophageal varices in Syria.

\section{Methods:}

102 cases at Damascus Hospital were retrospectivly in this study. The averages of the comparative independent groups were compared using parametric tests (T-Test Independent Samples, One way ANOVA) with the test for homogeneity to compare group variance and dimensional comparisons using LSD and Games-Howell

\section{Results:}

102 patient were included in the study, of them $66(65.3 \%)$ were males and $35(34.7 \%)$ were females and $52(51.5 \%)$ were smokers. Most of patient had complained from cirrhosis 46 (45.1\%), hemorrhagic vomiting 30 (29.4\%), and dark stools 18 (17.6\%). Esophageal varices were diagnosed by endoscopy 81 (79.4\%), and clinical examination $21(20.6 \%)$. Bleeding esophageal varices were treated using endoscopic ligation 38 (80.9\%), and injection histoacryl sclerotherapy $9(19.1 \%)$. The most common cause of cirrhosis was significantly viral hepatitis B ( $p$.).0.05>Esophageal varices degrees were significantly associated with smokers, diagnostic endoscopy, and endoscopic ligation $(p .(0.05>$

\section{Conclusion:}

More details have been identified regarding symptoms, risk factors, diagnosis, and treatment of esophageal varices in Syria. That leads to conduct better healthcare programs and stress the importance of regular health checkups. Furthermore, esophageal varices it was shown that had higher incidence rates among viral hepatitis B patients. Additional studies are required to determine management and to get better insight on the treatment

\section{Introduction And A Brief Review:}

\section{Anatomical introduction to the Digestive tract circulation:}

It is a group of veins that come from the digestive system and are parallel to the main arteries that supply the foregut, midgut and hindgut with perfusion, including the celiac, superior mesenteric, and inferior mesenteric arteries respectively. It forms a single venous passage to the liver. The abdominal vein carries waste from the foregut and stomach, through the second part of the duodenum, the superior mesenteric vein drains the third part of the duodenum through the first two-thirds of the transverse colon, finally, the inferior mesenteric vein drains the remaining one-third of the transverse colon through the rectum These veins carry nutrients and toxins from the digestive intake and ultimately provide approximately $75 \%$ of the liver's blood supply, the remainder coming from the hepatic artery, eventually draining into the systemic circulation [1]

\section{The function:}

What is absorbed by the small intestine and the right colon goes to the superior mesenteric vein

What is absorbed by the left colon goes to the inferior mesenteric vein. The inferior mesenteric vein drains into the splenic vein, which joins the superior mesenteric vein. They form the portal vein that enters the liver, and therefore all that is absorbed by the intestine enters the liver. The portal vein also receives drainage from the gallbladder, spleen, pancreas, and stomach. The portal vein enters the liver within the hepatoduodenal ligament, going posteriorly to the corresponding hepatic artery and the common bile duct. When the 
portal vein reaches the hilum of the liver, it divides into two branches: right, left, and left, then divides into more divisions and is fed by the hepatic sinusoids. The gradually smaller branches that come from the venous divisions form the portal veins. After the blood is mixed within the hepatic sinuses, it is collected within the terminal hepatic vein or the central vein, the intrahepatic and portal venous pressures are regulated by the portal venous sphincter. These vessels carry blood to the liver, as well as drain toxins and nutrients from the gut, then drain the blood into the inferior vena cava (IVC), and the remaining $25 \%$ of blood flow to the liver is via the corresponding hepatic artery. Hepatic parenchymal cells are among the most waste-rich cells in the entire human body. Oxygenated blood is fed from the hepatic artery and nutrient-rich blood from Visceral vessels Through the portal venous system, this blood mixes into the hepatic sinuses. These central veins then form to form the hepatic vein, which empties the liver back into the inferior vena cava, allowing blood to return to the heart and systemic circulation. A large volume of blood is present within the portal venous system and a similarly large volume of blood is present within the liver. The volume of blood in this sentence affects the body's response to fluid loss or excess. The blood within the portal vein is important for the functional role of the liver, including lipoprotein metabolism, drug metabolism, and detoxification, as well as endocrine homeostasis. [2]

\section{Movement of blood in the hepatic portal system:}

Normal portal circulation: This circulation is connected to the central circulation via the venules above the liver, which drains into the inferior vena cava, where after filtering the incoming sorbents To the liver through the portal vein and the manufacture of necessary elements for the body, these materials and elements are transmitted to the veins above the liver, and then to the inferior vena cava, which drains into the heart, so that the heart Pumping these substances all over the body Systemic portal venous anastomosis: there is a connection between the portal system and systemic veins Other ways than the main one. The most important of these are: Esophagus: between the left gastric vein (which drains into the portal vein) and the lower esophageal veins that drain To the individual sentence (and then to the superior hollow). Rectum: between the superior rectal veins (to the door) and the middle rectal veins and inferior (to the inferior hollow). Peri-umbilical: between the umbilical vein within the rounded ligament (to the door) and the veins of the abdominal wall. Anastomotic and clinically insignificant renal veins,. These anomalies are closed and neglected after fetal life and reopened in the event of overgrowth. Tension of the portal vein to try to secure a path to the systemic circulation instead of the path through the portal vein, and this results in a number of clinical signs that we will discuss later.

\section{Embryonic origin:}

Formation of the portal venous system begins during the fourth week of pregnancy and continues until the twelfth week. Selective includes the central part of the left vitelline vein and their anastomoses. Dorsal and ventral anastomoses lead to the main portal vein and left portal vein, respectively. [3]

\section{Surgical considerations::}

Knowledge of both normal and variable anatomy is of paramount importance, especially for surgery. Portal venous branching patterns are often evaluated prior to surgery via imaging techniques. Doppler ultrasound is the most useful imaging technique for identifying aneurysms or portal vein thrombosis. Helical CT is a valuable tool for visualizing the branching pattern of the portal venous system. Anatomical variants of portal vein branching are essential for imaging recognition of liver surgery to maintain perfusion of the remaining liver segments. In addition, portal venous anatomy is important for liver transplantation as both donor venous anatomy and recipient anatomy require analysis to ensure proper selection of donor and recipient. Portal vein thrombosis is a common occurrence in patients with hypercoagulable diseases, such as malignancy and cirrhosis, and can present a challenge at the time of liver transplantation. Due to the increased morbidity and mortality associated with this condition at the time of transplantation, this thrombotic condition is considered a relative contraindication for liver transplantation. Portal vein recanalization (PVR) and transjugular systemic portosystemic shunts (TIPS) are safe options for patients with portal vein thrombosis who are in urgent need of liver transplantation. Because of the large amount of blood inside the liver that flows through the portal system, patients with liver injuries are at increased risk of hemorrhagic shock and death due to blood loss. Pressure on the portal vein, bile artery, and bile duct, known as the Pringle maneuver, stops blood flow to the liver It allows a better view of the surgical field and helps prevent rotational trauma. [4]

\section{Introduction to esophageal varices:}

But before going into the introduction to the esophagus varices, some concepts must be clarified. Varicose definition: Varicose veins are enlarged, twisted veins that can occur anywhere on the body, but are most common in the legs. Varicose veins are not considered a serious medical condition, but they are It can be uncomfortable and can lead to more serious problems. [5] Introduction to 
Esophageal Rotation: The esophagus receives blood from many blood vessels, where the upper part of the esophagus and the upper esophageal sphincter receive blood from the lower thyroid artery. Blood from the left gastric artery and left inferior phrenic artery. Venous drainage also varies across the esophageal path, where the upper and middle parts of the esophagus drain into the veins of the individual and half of the individual, while the blood of the lower part drains into the left gastric vein. All previous veins drain into the superior vena cava except for the left gastric vein, which is a branch of the portal vein.叉 [6]

\section{Introduction to gastric rotation:}

There are three arteries that supply the great curvature: The upper part of the curvature: the short gastric arteries (originating from the splenic artery), and also feeding the fundus of the stomach. The gastro-omental vessels: Middle part of the curvature: gastric branches of the left gastro-omental artery. Bottom of the curvature: gastric branches of the right gastro-omental artery. There are two arteries that supply the small curvature: Top of the curvature: the left gastric artery, which also supplies the heart area. The lower part of the curvature: the right gastric artery, The veins in the stomach run parallel to the arteries, the right and left gastric veins drain into the hepatic portal vein. The short gastric vein, left and right gastro-omental veins eventually drain into the superior mesenteric vein. [7] Esophageal varices:

They are enlarged, dilated veins located under the esophageal mucosa, and they are abnormal veins They often arise due to a blockage in blood flow through the portal vein, which carries blood from the intestine, pancreas, and spleen to the liver, which appears under the mucosa of both the stomach and esophagus. It also links the portal and systemic circulation, when portal hypertension occurs (the most common result of cirrhosis of the liver), resistance to portal blood flow, and increased portal venous blood flow, where the portal venous pressure is less than $5 \mathrm{mmHg}$ and we note that it is elevated when it exceeds 13 millimeters of mercury, given that the circulation of the portal vein is more than $1500 \mathrm{ml} / \mathrm{min}$ If there is a blockage, this leads to high blood pressure in the portal vein. The body's response to increased venous pressure is the development of anastomoses. These systemic portal anastomoses divert blood from the portal venous system to the inferior and superior vena cava. At the same time, an important system is the gastro-esophageal anastomosis that leads to the development of esophageal varices, when these veins enlarge and expand and form We have varicose veins, and when they rupture (the most common complication) they produce severe bleeding. Bleeding from esophageal varices is the third most common cause of upper GI bleeding, after duodenal and gastric ulcers [8] [9] [10] [11][12]

\section{Causes of portal hypertension:}

Pre-hepatic: Portal vein occlusion (EHPVO) by thrombus, tumor, or large splenomegaly with increased blood flow into the splenic vein. Post-hepatic: Acute right-sided heart failure, pericarditis and hepatic vein obstruction (Budd-Chiari syndrome). intrahepatic: Cirrhosis accounts for most cases of portal hypertension. Other rare causes of portal hypertension include: Schistosomiasis - Sarcoidosis Wilson's disease - tuberculosis - lipid disorders - alpha-1 antitrypsin deficiency - Primary biliary cirrhosis - Hereditary hemorrhagic telangiectasia [13]

\section{Incidence:}

At diagnosis, $30 \%$ of patients with cirrhosis have varicose veins that rise to $90 \%$ within 10 years. The rate of variceal bleeding during the first year is $5 \%$ for small varicose veins, $15 \%$ for large varicose veins.Portal hypertension is common in children with chronic liver disease (CLD). [14]

\section{Prevalence :}

It is more common in males than in females. Fifty percent of patients with esophageal varices will experience bleeding at some point. The mortality rate for variceal bleeding is $10-20 \%$ in the six weeks after the bleeding occurs. The two common causes of portal hypertension in the West are alcohol and viral hepatitis. In Asia and Africa, the most common causes of portal hypertension include schistosomiasis and hepatitis $B$ and $C$, varying by geographic region. [14]

\section{The pathophysiology of esophageal varices:}

Portal hypertension causes the development of a systemic portal anastomosis, in order to lower the pressure of the portal circulation. Normal portal pressure ranges from $5-10 \mathrm{mmHg}$ but in the presence of portal vein obstruction, the pressure may be as high as $15-20$ $\mathrm{mmHg}$. Since the portal venous system does not contain valves, resistance at any level between the visceral vessels and the right side of the heart results in an increase in pressure and return blood volume. The anastomoses expand slowly and the systemic circulation 
is connected to the portal venous system. Over time, this leads to engorgement of the submucosal venous plexus with dilated tortuous veins in the distal esophagus. Varicose rupture causes bleeding.[15]

\section{Pathophysiology of portal hypertension:}

Increased resistance to portal vein flow at the level of the hepatic sinusoids is caused by: Intrahepatic vasoconstriction due to decreased production of nitric oxide, and increased release of endothelin-1 (ET-1), angiotensinogen, and eicosanoids. Sinusoidal remodeling disrupts blood flow. Increased portal vein blood flow caused by hyperdynamic circulation due to dilatation of visceral arterial vessels through mediating agents such as nitric oxide, prostacyclin and tumor necrosis factor.[16]

\section{Risk factors for varicose bleeding:}

The size of the varicose veins, as the larger the size of the varicose veins, the greater the possibility of rupture. The advanced classification of pediatric insufficiency also increases the risk of bleeding. The presence of red markings on the varicose veins during endoscopy is also associated with the possibility of rupture. Chronic cough and constipation. Active alcohol consumption [17] .

\section{Symptoms and diagnosis:}

Often the first indication of varicose veins is an episode of gastrointestinal (GI) bleeding: Hematemesis, lower GI bleeding or tarry stools, and occult bleeding (anaemia) is uncommon. [18]

\section{Disease history and symptoms:}

Variceal bleeding can be the initial symptom of previously undiagnosed cirrhosis. In the clinical history we may find the following findings: Alcoholism and exposure to blood-borne viruses.

Hematemesis, tarry stools, or haemorrhage, mass upper gastrointestinal bleeding can occur. Jaundice, Weight loss in patients with chronic liver failure, Loss of appetite, Abdominal discomfort, Itch, Symptoms of encephalopathy - a change in mental status, Hepatic nephropathy, Muscle cramps. [19]

\section{Clinical examination:}

Assessment of hemodynamic stability: hypotension, tachycardia (active bleeding).

Abdominal examination: Liver size (the liver is enlarged, remains normal, or becomes smaller, depending on the cause of cirrhosis). [20]

The degree of spleen enlargement: the return of blood through the splenic vein (forming the portal vein) as a result of an obstruction in the portal vein. [21] The return of blood to the spleen leads to an increase in its size and enlargement, which is often associated with hypersplenism (hyperfunction of the spleen), and since the spleen is a graveyard for red blood cells, eggs and platelets, therefore, in the case of hypersplenism, it will eat them and destroy them more, resulting in: Anemia; caused by a lack of red blood cells, Leukopenia: This causes infections and immunodeficiency, Hemorrhage: As a result of a lack of platelets.

\section{Treatment:}

In the event of a deterioration in the blood count, we resort to removing the spleen Cirrhosis patients had an enlarged spleen, ranging from 35 to $50 \%$ of cases. The degrees of spleen enlargement are classified into: Normal is less than $11 \mathrm{~cm}$. Moderate spleen if the largest dimension of the spleen ranges between $11-20 \mathrm{~cm}$. Severe spleen if the spleen has a larger dimension greater than $20 \mathrm{~cm}$. Degree of love: three degrees, namely: [22] Grade 1: Mild visible only on ultrasound or computed tomography. Grade 2: detectable by clinical examination. Grade 3: Visually evident.

\section{Hemorrhoidal varicose:}

Reason: blood moves as a result of opening the anastomosis from the upper rectal vein (connected to the portal system) to the middle and lower rectal veins (hemorrhoidal) (connected to the systemic circulation via the inferior vena cava), which leads to severe congestion in the hemorrhoidal veins and the formation of what looks like hemorrhoids. Peripheral scars of alcohol abuse, Spider angioma on the chest / back, Gynecomastia, rest erythema, Testicular atrophy, Hepatic encephalopathy. [23]

\section{Screening and Evaluation:}


Initial tests (laboratory, radiological), Anemia: Hemoglobin may be normal in active bleeding and may take six to 24 hours for its value to decrease. Other causes of anemia are common in cases of cirrhosis. Thrombocytopenia is the most sensitive and specific laboratory marker associated with portal hypertension and large esophageal varices. Elevated aspartate aminotransferase (AST), alanine aminotransferase (ALT), alkaline phosphatase, bilirubin; Prolonged prothrombin time (PT), decreased albumin to cirrhosis, BUN is often elevated in GI bleeding, and sodium may be decreased in patients treated with terlipressin. The following should also be investigated: Study of the thrombotic state, Kidney function investigation, Arterial blood gases, Hepatitis antibodies.[24]

\section{Esophagogastroduodenoscopy:}

It can identify active bleeding varicose veins as well as large varicose veins resulting from recent bleeding. It can be used to treat bleeding with esophageal ligation (sclerotherapy is preferred over ligation); prevention of rebleeding; detection of gastric varices, portal gastropathy of hypertension; Diagnostics of alternate bleeding sites Can identify and treat non-bleeding varicose veins (prominent submucosal veins in the distal third of the esophagus) [25]. The upper gastrointestinal endoscopy can also determine the degree of esophageal varices Mild: straight or slightly twisted esophageal varices without bleeding signs. Moderate: esophageal varices that are straight or slightly twisted, with hemorrhagic signs, or esophageal varices with twisted but no hemorrhagic signs. Severe: esophageal varices that are twisted or twisted, and have hemorrhagic signs, or esophageal varices that are cherry-like or nodular, regardless of the presence of hemorrhagic signs or not. [26]

\section{Other actions:}

Transient elastic imaging (TE) to identify patients with chronic cirrhosis at risk for clinically significant portal hypertension (CSPH). Hepatic vein pressure gradient (HVPG) greater than $10 \mathrm{mmHg}$ is the gold standard for the diagnosis of clinically significant portal hypertension (CSPH) (normal: $1 \mathrm{mmHg}$ to $5 \mathrm{mmHg}$ ), which may determine a hepatic vein pressure gradient (HVPG) response equal to or $>10 \%$, less than, or equal to $12 \mathrm{mmHg}$ for intravenous propranolol and non-selective beta-blocker (NSBB) responders are associated with a significantly reduced risk of variceal bleeding. Video endoscopy may be an alternative to traditional endoscopy. Doppler sonography (second line): showing the diameter and flow of the portal vein, spleen, and anastomoses; With good sensitivity to gastric varices, follow-up after ligation or transjugular systemic intrahepatic portosystemic venous shunt (TIPS) is helpful. CT or MRI angiography (second line, not routine): Shows channels of large blood vessels in the abdomen and mediastinum; Demonstrates blood flow through the intrahepatic portal vein and splenic vein. Abdominal arteriography in the venous phase: showing the portal vein and anastomoses; Diagnosing hepatic vein obstruction, measuring portal vein pressure using a catheter in the retrograde vein in the hepatic vein, abdominal ultrasound may reveal blockage of the bile ducts (for example, due to cancer).[27]

\section{management and treatment:}

Chronic diseases associated with cirrhosis must be treated. Hepatic encephalopathy and sepsis often complicate variceal bleeding [28] active bleeding: Open intravenous (IV), resuscitation of blood circulation Excessive blood transfusion increases portal vein pressure. The risk of rebleeding increases by treating blood clots when necessary. Fresh frozen plasma may increase blood volume and increase the risk of rebleeding. Monitor mental status. Severely avoid anesthesia, nephrotoxic drugs, and beta-blockers. IV octreotide to lower portal venous pressure as an adjunct to endoscopic management. An intravenous dose of 50 mcg followed by an instillation of $50 \mathrm{mcg} /$ hour. Terlipressin (alternative): $2 \mathrm{mg} \mathrm{IV} \mathrm{every} 4$ hours for 24 to 48 hours, then $1 \mathrm{mg}$ every 4 hours Erythromycin $250 \mathrm{mg}$ IV 30 to 120 minutes before endoscopy, also indicated for antibiotic prophylaxis with oral norfloxacin $400 \mathrm{mg}$ or intravenous ceftriaxone, $1 \mathrm{~g}$ every 24 hours for up to 1 week, urgent upper Gl endoscopy for diagnosis Treatment If contraindicated, start with betablockers (nitrates alternative), varicose ligation is preferred over sclerotherapy for bleeding varicose veins and for medium to large non-hemorrhagic varicose veins to reduce the risk of bleeding. For ligation lower rates of rebleeding, fewer complications, faster cessation of bleeding, and higher rate of varicocelectomy, repeat ligation or crucifixion if bleeding recurs. If endoscopic therapy fails, consider self-expanding esophageal metal stents or oral Sengstaken-Blakemore tube placement for up to 24 hours to stabilize the patient for TIPS. [28] [29]

\section{treatment complications:}

Transient dysphagia (73\%), chest pain (65\%), esophageal ulcers (63\%), ulcerative bleeding (14\%), post-treatment bleeding (13\%), esophageal stricture (10\%), pleural effusion (9)\% (, pericarditis $(0.4 \%)$ and esophageal perforation ( $0.4 \%)$, up to two thirds of patients with bleeding varices develop infection (most commonly spontaneous bacterial peritonitis, urinary tract infection, pneumonia). Active bleeding beta-blockers, which lower blood pressure and reduce the physiological increase in heart rate during acute bleeding, should be avoided. [30] 
Prevent recurrence of severe bleeding: Vasoconstrictors: terlipressin, octreotide (reducing portal vein pressure). Non-selective betablocker (NSBB). Endoscopic varicose vein ligation (EBL): If bleeding recurs or portal pressure measurement shows portal pressure still greater than $12 \mathrm{mmHg}$. Tips: Second-line treatment if the above methods fail; A transjugular systemic intrahepatic portal shunt (TIPS) reduces portal pressure by creating a connection between the hepatic vein and the branch of the intrahepatic portal vein [31] [32]

\section{The main treatment:}

first line; a non-selective beta-blocker (NSBB) reduces portal vein pressure and reduces the risk of first bleeding from $25-15 \%$ in primary prevention.

Carvedilol: $6.25 \mathrm{mg}$ daily is more effective than a non-selective beta-blocker (NSBB) (propranolol and nadolol) in projecting the hepatic vein pressure gradient (HVPG). Chronic prevention of rebleeding (secondary prevention): a non-selective beta-blocker (NSBB) and endoscopic variceal ligation (EBL) reduce the rate of rebleeding to a similar extent, but beta-blockers reduce mortality, whereas ligation does not. It is also not contraindicated in patients waiting for a liver transplant. Conservative treatment is used in low grades of varicose veins $[33,34]$. The second line (the least invasive): Ligation of esophageal varices During ligation, proton pump inhibitors, such as lansoprazole $30 \mathrm{mg} /$ day, are used until the varicose veins are hidden. Management of Budd-Chiari syndrome:

anticoagulation, angioplasty, thrombolysis, transjugular systemic intrahepatic portosystemic shunt (TIPS), and heterologous liver transplantation. Management of extrahepatic portal vein occlusion: bypassing the left mesenteric portal vein (Meso-Rex procedure). Re-endoscopy, liver transplantation, interventional radiology, and transjugular systemic intrahepatic portosystemic shunt (TIPS). The pneumococcal and hepatitis B vaccine (HAV-HBV) should be considered.[35]

\section{Surgical (invasive) treatment:}

(TIPS) Transjugular Intrahepatic Portosystemic Shunt : It is a salvage procedure to stop acute variceal bleeding. However, the procedure is also associated with serious complications including encephalopathy and shunt obstruction within 12 months. TIPS may be a precursor to a liver transplant. Objective: To establish a stent connection between the portal circulation on one side (the portal vein) and the systemic circulation on the other hand (the hepatic vein) through the liver, in order to reduce pressure in the portal system and stop the bleeding of varicose veins. The catheter with a balloon is inserted using a guide through the internal jugular vein and then the Inferior vena cava, then hepatic veins, then entering the hepatic parenchyma to reach the portal vein. Within the hepatic parenchyma, a duct is formed that connects the portal vein to one of the liver veins, to Then put the link (Stent) We rarely use this method, as in the case of unresponsiveness to endoscopic treatments.[36]

Distal Splenorenal Shunt: It is based on the anastomosis of the splenic vein with the left renal vein, and blood moves from the varicose veins to the splenic vein and from it to the left renal vein and from it to the inferior vena cava. It aims to treat portal hypertonia and reduce the risk of bleeding. The spleen can be removed to relieve symptoms of hypersplenism. However, the surgical method is rarely used. [37]

Esophagectomy: In rare cases of uncontrollable bleeding.

\section{Hospital discharge criteria:}

The bleeding has stopped, Stabilization of blood circulation, Develop an appropriate plan for the treatment of comorbidities.[38]

\section{differential diagnosis:}

Acute gastric erosions and abrasions, Duodenal ulcer, ectoderm, stomach cancer, Mallory-Weiss syndrome, Nasogastric tube trauma, Portal gastropathy due to high blood pressure.[23]

\section{warning:}

Once a patient has had one episode of variceal bleeding, there is a $70 \%$ chance of recurrence, and at least $30 \%$ of rebleeding episodes are fatal. Most deaths occur within the first few days after bleeding. Mortality rates are highest in the presence of surgical intervention and acute variceal bleeding. In the case of cirrhosis, the one-year survival rate is $50 \%$ for those who survive 2 weeks after variceal bleeding. In-hospital mortality remains high due to the severity of cirrhosis. Diagnosis in portal hypertension not associated with cirrhosis is the best prognosis.[39]

\section{Complications:}


Early complications: Inhalation, Multiple member failure, esophageal perforation.

Hemorrhagic shock and death.[40]

Late complications: Contagious varices are after excision of esophageal varices, Esophageal varices can degenerate, Hepatic encephalopathy, Kidney failure, Hepatorenal Syndrome, Infections after varicose veins ligation.[41]

\section{Miscellaneous tips::}

Endoscopic ligation of the varicose veins should be repeated every 1 to 4 weeks until the varicose veins are removed, or if a TIPS is performed, repeat the endoscopy to assess for bleeding again. Endoscopic examination in patients with known cirrhosis should be performed every two to three years and annually in patients with decompensated cirrhosis. Patients with cirrhosis and platelet count greater than 150,000 can avoid endoscopy and can be followed up by platelet count [31]

\section{Study Aims:}

Our study aims to determine the methods used in the treatment of esophageal varices. Linking esophageal varices risk factors with some variables. Discovering the methods currently used in Syria with a comparison with the methods used outside it.

\section{Materials And Method:}

A retrospective study was conducted. The hospital's approval was obtained to review patients' data, starting in Jan 2018 to Dec 2021. Samples were collected from the internal digestive, general surgery, vascular and thoracic departments in the hospital. The questionnaire was created in an automated online Google form. The data has been archived by Microsoft Excel 2019. The study included 102 patients admitted to the Gastrointestinal Division for the management and treatment of esophageal varices, either endoscopy or referral to the Department of Surgery at Al-Mujtahid Hospital in Damascus. Below we review some characteristics of the sample according to demographic variables and the variables that measure the incidence of esophageal varices. A pilot study was conducted on the first sixteen patients of the study to evaluate the questionnaire and its efficiency, and some modifications were made to the structure of the initial questionnaire afterwards.

\section{exclusion criteria}

Archiving errors, Cases misdiagnosed as esophageal varices, Outpatient cases accepted in the upper GI endoscopy department that do not contain patient data, Cases lack data in a way that cannot be studied.

\section{statistical analysis:}

The statistical study was conducted using SPSS software. The demographic characteristics were described, and the sample distribution according to the study variables was described. The Chi-Square independence test was also used to test the independence of qualitative variables, and correlation tests according to the Krams coefficient to study the relationship between qualitative variables, and Spearman's coefficient to study the relationship between quantitative variables. The averages of the comparative independent groups were compared using parametric tests (T-Test Independent Samples, One way ANOVA) with the test for homogeneity to compare group variance and dimensional comparisons using LSD and Games-Howell.

\section{Results:}

\section{demographic statistical indicators:}

We note that the ages of patients between 14 and 80 years with an average of 53.11 years

Approximately two thirds of the sample are males, or $65.3 \%$ of patients, compared to a third of the sample are females, representing $34.7 \%$ of patients.

The majority of the sample was married, with a percentage of $93.7 \%$ of patients, while singles accounted for $6.3 \%$ of patients. All members of the sample are holders of Syrian Arab nationality, ie $100 \%$ of them. Approximately one-fifth of the sample, or $22 \%$ of the patients, live in Damascus, a quarter of the sample, or $25.3 \%$ of them live in Damascus countryside, $8.8 \%$ of them live in Aleppo and 
the same in Homs and Deir ez-Zor, 3.3\% of them live in Hama, 4.4\% of them live in Daraa and the same in Tartous, $2.2 \%$ of them live in As-Suwayda and the same in Al-Hasakah, $1.1 \%$ of them live in Lattakia and Quneitra, $7.7 \%$ of patients live in Raqqa, and there are no patients who live in Idlib within the sample. The lifestyle included both smoking and alcohol, and the following table shows the distribution of the sample. Nearly half of the sample, $51.5 \%$ of smokers, compared to $48.5 \%$ of the sample are non-smokers.

The largest proportion of the sample, that is, $91.2 \%$ of the respondents do not drink alcoholic beverages, compared to $8.8 \%$ of them drink alcoholic beverages.

\section{main complaint:}

$45.1 \%$ of patients were hospitalized due to cirrhosis of the liver, $29.4 \%$ suffered from vomiting blood, $17.6 \%$ of patients were hospitalized due to the presence of tar stools, $3 \%$ of patients experienced sudden pain in the abdomen and similarly suffered from gastrointestinal bleeding and alterations in consciousness, $1 \%$ Some of them were referred to the hospital because of the large size of the abdomen. The majority of patients, $91.2 \%$ of them suffer from cirrhosis of the liver, compared to $8.8 \%$ of them without it.

\section{Clinical and radiographic examination:}

from the results of the clinical examination (clinical findings) We note that: More than a quarter of the sample, or $27.2 \%$ of patients, have jaundice. $29.6 \%$ of patients are infected with Ascites. One third of the sample, or $33.1 \%$, suffer from anemia. $2.4 \%$ of them have hemorrhoids. $7.7 \%$ of the sample members gave the results of their clinical examination that they are normal.

We note from the results of the radiographic examination (the degree of spleen and liver enlargement) that: $18.6 \%$ of patients had mild splenomegaly, $33.3 \%$ had moderate splenomegaly, $24.5 \%$ had large splenomes, $2 \%$ had their spleen removed for other reasons, compared to $21.6 \%$, or nearly one-fifth of the sample. They have an enlarged spleen. $36.3 \%$ of patients had mild hepatomegaly, $7.8 \%$ had moderate hepatomegaly, $2 \%$ had large hepatomegaly, compared to $29.4 \%$ of them had small liver size, and $24.5 \%$ or nearly a quarter of the sample did not. They have an enlarged liver. $14.7 \%$ of patients have esophageal varices of the first degree, $27.5 \%$, or more than a quarter of the sample, have esophageal varices of the second degree, $39.2 \%$ of them, or more than a third of the sample, have esophageal varices of the third degree, only $3.9 \%$ of patients have Esophageal varices of the fourth degree, compared to $14.7 \%$ of the sample without esophageal varices and they are 15 people. $18.6 \%$ of patients have mild ascites (ascites of the abdomen), $26.5 \%$ of them have moderate degree of ascites, $25.5 \%$ of them, or nearly a quarter of the sample, have severe ascites, compared to $29.4 \%$ of patients who do not have ascites.

\section{Concerning the type of liver damage and its antibodies, we note that:}

$48.9 \%$ of patients had a positive hepatitis antibody test, while $51.1 \%$ had a negative hepatitis antibody test. $1.1 \%$ of patients were infected with hepatitis A, $37.9 \%$ of them were infected with hepatitis B, $12.6 \%$ of them were infected with hepatitis C, and there were no patients with hepatitis $D$ in the sample, $3.4 \%$ of them were infected with Wilson's disease, $4.6 \%$ of them They had autoimmune hepatitis, $1.1 \%$ of them had fatty liver, $4.6 \%$ had alcoholic hepatitis, compared to $34.5 \%$ of patients with idiopathic or under study. The lower and upper bounds, the arithmetic mean and the standard deviation of the medical test results were also calculated. The following table illustrates this. The level of sodium in the blood (NA) in the patients ranged between 123 and 148 , with an average of 136.89 , but the values of the analyzes as a whole may deviate from the mean by $4.531 \pm$. The level of potassium in the blood (K) of patients ranged between 2.9 and 133 with an average of 5.769, although the values of the analyzes as a whole may deviate from the mean by $14.3285 \pm$. The erythrocyte sedimentation rates or sed rate (ESR) in patients ranged between 2 and 140 with an average of 40.98 , although the values of the analyzes as a whole may deviate from the mean by $34.3445 \pm$. The values of platelet analysis (PLT) in patients ranged between 1.9112000 with an average of 2794.85 , however the values of the tests in total may deviate from the mean by $13954.92 \pm$. The percentages of hemoglobin in the blood (HB) in the patients ranged between 5.6 and 14.5 with an average of 9.524, although the values of the analyzes as a whole may deviate from the mean by $2.2034 \pm$. The result of the prothrombin time (PT) test for patients ranged between 11 and 100, with an average of 55,417, but the overall results may deviate from the mean by \pm 22 . The values of analysis of white blood cell count (WBC) in patients ranged between 1.9 and 34,000, with an average of 5663.912, although the values of the analyzes as a whole may deviate from the mean by $6687.6592 \pm$. The values of gammaglutamyltransferase (GGT) analysis in patients ranged between 7 and 254, with a mean of 65,765, but the overall analyzes values may deviate from the mean by $54.4 \pm$. The values of the analysis of the level of alkaline phosphatase (ALP) in patients ranged between 7 and 603 with an average of 151.336, although the values of the analyzes as a whole may deviate from the mean by \pm 110 . The values of the analysis of the level of alanine aminotransferase (ALT) in patients ranged between 5 and 468 , with a mean of 44.78, but the values of the analyzes as a whole may deviate from the mean by $63.04 \pm$. The values of aspartate transaminase (AST) 
enzyme level analysis in patients ranged between 8 and 390, with a mean of 61.5 , but the values of the tests as a whole may deviate from the mean by \pm 60.903 .

\section{Diagnostic and treatment modalities:}

Diagnostic methods included (upper gastrointestinal endoscopy, computed tomography, echo-Doppler and previously diagnosed cirrhosis). The treatment modalities were either a review of previous cirrhosis without any symptoms and included the research (medications to reduce portal vein pressure, endoscopic such as stapling, endoscopic such as sclerotherapy with injection of histoacryl, endoscopically with injection of adrenaline) or transverse bleeding and included (endoscopic such as ligation with clips, endoscopic such as injection sclerotherapy) Histoacryl, pharmacologically used to slow the flow of blood, was mounted in the jugular systemic portal and pressed against the varicose veins with a balloon). We note that the majority of patients in the sample were diagnosed with varicose veins by axial gastrointestinal endoscopy, and their percentage was $79.4 \%, 20.6 \%$, or nearly a quarter of the sample were previously diagnosed and then varicose veins were investigated by endoscopy, and there are no patients whose disease was diagnosed by CT or Echo Doppler. As for the methods of treating esophageal varices, $39.2 \%$ of the sample were not symptomatic (40 patients), compared to $46.1 \%$ who had bleeding symptoms (47 patients). Distribution of patients without symptoms (coincidence or investigations following the discovery of cirrhosis) according to treatment modalities: $38.2 \%$ were treated endoscopically, such as clip ligation, 3.6\% were treated endoscopically, such as sclerotherapy with histoacryl injection, $1.8 \%$ were treated endoscopically with epinephrine injection, and 29.1 were treated endoscopically such as sclerotherapy. The presence of patients who were treated with drugs to lower the portal vein pressure in the sample. Patients who showed symptoms of bleeding according to treatment modalities were distributed to $80.9 \%$ who were treated endoscopically, such as ligation with clips, and $19.1 \%$ were treated endoscopically, such as sclerotherapy with histoacryl injections.

\section{Second, the inferential analysis}

Testing the relationship between the degree of varicose veins and some study variables

First: The relationship between the degree of esophageal varices and gender:

the absence of esophageal varices was greater in males, where the percentage amounted to $10.9 \%$ of the sample compared to $4 \%$ of females. Also, esophageal varices of the fourth, third and second degree are more present in males, and therefore the gender change did not make a difference in the presence and degree of esophageal varices. To confirm this, a Chi-Square test for independence was conducted and the previous results appeared.

We note that esophageal varices of the second and third degree are more present in the places where the relationship is established between them. An independence test was conducted and the following results were obtained. We note the probability value of Pearson Chi-Square is less than 0.05 hypothesizing the null hypothesis that says that the degree of esophageal varices and smoking are independent; correlation between them. And in order to find out the relationship between them, the coefficient of the variables was reached and the following results were found.

We note that the presence and degree of esophageal varices was not affected by the presence of alcohol, as the percentages were generally greater for individuals who do not drink alcoholic beverages, noting that individuals who drink alcohol accounted for only $8.8 \%$ within the sample. To study the relationship between them, the Chi-Square test for independence was conducted, and the following results appeared. We note that the $p$ value of Pearson Chi-Square is greater than 0.05 and therefore we accept the null hypothesis that the degree of esophageal varices and alcohol consumption are independent; That is, there is no correlation between them and therefore any difference in the presence of esophageal varices and drinking alcohol was not significant.

\section{The relationship between the degree of esophageal varices and cirrhosis of the liver:}

We note that the presence and degree of esophageal varices was not affected by cirrhosis, as the percentages were generally greater for individuals with cirrhosis, noting that individuals without cirrhosis accounted for only $7.8 \%$ of the sample. To confirm this, a ChiSquare test for independence was conducted and the following results appeared. We note that the $p$ value of Pearson Chi-Square is greater than 0.05 and therefore we accept the null hypothesis which says that the degree of esophageal varices and cirrhosis are independent; That is, there is no correlation between them, and therefore any difference in the presence of esophageal varices with cirrhosis of the liver was not significant.

Page $10 / 15$ 
We note that the largest percentage of patients with esophageal varices of the first and third degree were infected with idiopathic hepatitis ( $8 \%$ and $20.7 \%$, respectively). The largest proportion of patients with esophageal varices of the second degree were infected with hepatitis B (12.6\% of the sample). As well as the largest proportion of individuals without esophageal varices were infected with hepatitis B (6.9\% of the sample). To study the existence of a relationship, the Chi-Square test for independence was conducted, and the following results appeared. We note that the $p$ value of Pearson Chi-Square is greater than 0.05 and therefore we accept the null hypothesis which says that the degree of esophageal varices and the type of hepatitis are independent; That is, there is no correlation between them and therefore any difference between them was not significant.

\section{The relationship between the degree of esophageal varices and the method of diagnosis:}

We note the largest proportion of patients with esophageal varices of various degrees were diagnosed with a higher endoscopy, people without esophageal varices, the largest proportion of them were diagnosed with clinical design with blood. To study the existence of a relationship, an independence test was conducted and the following results were obtained. We note the probability value of Pearson Chi-Square is less than 0.05 hypothesizing the null hypothesis that says that the degree of esophageal varices and the method of diagnosis are independent; relationship between them. In order to find out the relationship between them, the coefficient of covariance was reached and the following results were found.

\section{The relationship between the degree of esophageal varices and the method of treatment (accidental):}

We note that the largest percentage of esophageal varices patients who had first, second and third degree bleeding symptoms were treated endoscopically, such as ligation with clips. To study the existence of a relationship, the Chi-Square test for independence was conducted, and the following results appeared. The table above shows that the probability value is less than 0.05 , meaning that at the $95 \%$ confidence level there is a statistically significant correlation between the degree of esophageal varices and the treatment method (transverse bleeding) and since the value of Kramer's coefficient was 0.441 , the relationship is medium between them.

\section{The relationship between sex and cirrhosis of the liver:}

The following table shows the distribution of patients' sample by gender and cirrhosis.

We note that the largest percentage of males suffer from cirrhosis of the liver, as well as females. Therefore, it can be observed that there is no relationship between them and to confirm this, the Chi-Square test was conducted to test the independence and the following results appeared. We note that the $p$ value of Pearson Chi-Square is greater than 0.05 and therefore we accept the null hypothesis which says that gender and cirrhosis are independent; That is, there is no correlation between them and therefore any difference between them was not significant.

\section{The relationship between cirrhosis of the liver and the type of hepatitis:}

We note that cirrhosis of the liver is associated with hepatitis B more, followed by idiopathic hepatitis, hepatitis C, autoimmune and alcoholic, and then Wilson's disease. To study the existence of a relationship, the Chi-Square test for independence was conducted, and the following results appeared. The table above shows that the probability value is less than 0.05 , meaning that at the $95 \%$ confidence level there is a statistically significant correlation between the type of hepatitis and cirrhosis, and since the value of Kramer's coefficient was 0.522 , the relationship is medium between them.

\section{The relationship between cirrhosis of the liver and the presence of esophageal varices:}

We note that the largest percentage of the sample is associated with the presence of esophageal varices with cirrhosis of the liver. To study the existence of a relationship, the Chi-Square test for independence was conducted, and the following results appeared We note that the Pearson Chi-Square $p$ value is greater than 0.05 and therefore accept the null hypothesis that the presence of esophageal varices and cirrhosis are independent; That is, there is no correlation between them and therefore any difference between them was not significant. We note that the rate of varicose veins in patients with cirrhosis is $77.5 \%$.

The relationship between cirrhosis of the liver and esophageal varices and the type of hepatitis: 
We note that the largest percentage of patients with esophageal varices and cirrhosis were infected with hepatitis $B$ and hepatitis of unknown cause. To study the existence of a relationship between them, the Chi-Square test for independence was conducted, and the following results appeared. The table above shows that the probability value is less than 0.05 , meaning that at the $95 \%$ confidence level there is a statistically significant correlation between the type of hepatitis and cirrhosis of the liver in the absence of esophageal varices, and since the value of Kramer's coefficient was 1, the relationship is complete.

\section{The relationship between the type of inflammation and the degree of hepatomegaly:}

The following table shows the distribution of the type of hepatitis and the degree of hepatomegaly. The random distribution of the proportions shows that there is no relationship between the type of hepatitis and the degree of hepatomegaly. To confirm this, a ChiSquare test for independence was conducted, and the following results appeared

We note that in the presence of esophageal varices, the Pearson Chi-Square $p$ value is greater than 0.05 and therefore we accept the null hypothesis that the type of hepatitis and the degree of hepatomegaly are independent; That is, there is no correlation between them and therefore any difference between them was not significant.

\section{Discussion:}

The study was conducted on a sample of 102 cases that met the patient exclusion criteria, and the study was of a regressive type. In this paragraph, we will discuss the statistical results we obtained and interpret them according to the data we have and compare the findings of our study with the results of other international studies conducted on the same subject. We will first discuss the results with statistical evidence and then we will quickly discuss the results that are not statistically significant and try to interpret these results. Before starting this, we summarize the statistically significant results in the previous paragraph as follows:

1. The degree of esophageal varices and smoking

2. Esophageal varices of various degrees were diagnosed with upper gastrointestinal endoscopy.

3. The first, second and third degree were treated endoscopically, such as ligation with clips. As for fourth degree esophageal varices patients, the largest proportion of them were treated endoscopically, such as sclerotherapy by injection of Histoacryl.

4. Cirrhosis of the liver with hepatitis B.

5. Statistical significance between cirrhosis of the liver in the absence of esophageal varices.

While we did not find a relationship between sex with cirrhosis of the liver, and between the degree of varicose veins and gender and the degree of varicose veins was not affected by cirrhosis of the liver.

Smokers were $21.8 \%$ more associated with the third degree of esophageal varices compared with non-smokers. Smoking is a major risk factor for esophageal varices, as indicated by recent studies [42] The upper gastrointestinal endoscopy is one of the most important diagnostic methods used, as it is used to detect various degrees of esophageal varices. In our study, the results showed that most patients who had symptoms of cirrhosis of the liver were diagnosed with third-degree esophageal varices by means of upper gastrointestinal endoscopy $38.2 \%$, compared to $38.2 \%$ With those diagnosed previously clinically and laboratory. Our study agrees with another study conducted in China that upper GI endoscopy is the best way to investigate moderate to severe esophageal varices. [43] The treatment of esophageal varices often depends on banding and placing clips, and it is also possible to use sclerotherapy by injecting histoacryl. Theoretically, the method of treatment, such as clipping, was more associated with third-degree esophageal varices, $53.2 \%$, compared to the endoscopic treatment such as sclerotherapy with Histoacryl injections. The latest studies recommend that the ideal method for the treatment of varicose veins of the third and fourth degree is by endoscopy with ligation endoscopic variceal ligation (EVL). [44] It was found through our study that the most important cause in Syria for cirrhosis of the liver is hepatitis B viral hepatitis $37.9 \%$ compared to hepatitis A, C, D, idiopathic, Wilson's disease, autoimmune, steatosis, and alcoholic. A study conducted on 1281 patients in China showed that $73 \%$ of the patients had antibodies to HBV. This suggests the high prevalence of HBV in Asia..[46][45] Patients who had cirrhosis but were not diagnosed with esophageal varices were $5.88 \%$ more associated with

Page $12 / 15$ 
hepatitis B than patients without cirrhosis and who did not have esophageal varices. This corresponds to the fact that not every patient with hepatitis B will develop esophageal varices. [47]

\section{Recommendation:}

Urging the medical staff to take a detailed clinical story about each patient's condition. Carrying out all required analyzes for patients of the gastrointestinal tract. Using modern classifications to assess the degree of esophageal varices. Educating the community about the necessity of adhering to a healthy diet, avoiding smoking, avoiding alcohol, general hygiene, and the use of sterilized shaving tools. Conducting additional studies to determine the presence of any complications following the measures taken for patients.

\section{Difficulties and limitations of the study:}

The sample size is limited due to the lack of information in the records and their poor organization. The difficulty of obtaining sufficient information about patients admitted to the hospital due to their lack of knowledge about their medical history and previous medical procedures. Difficulty communicating with patients outside the hospital and following up on continuous monitoring of them. Not to put complications or follow-up in the records. Insufficient number of records are given by employees. Not to perform surgical interventions on esophageal varices.

\section{Declarations}

\section{Ethical approval and consent to participate:}

The study protocol was approved by the Research Ethics Committee in Syrian Private Universities, and the ethical committees in the concerned hospital. All procedures performed in studies involving human participants were in accordance with the ethical standards of the institutional and/or national research committee and with the 1964 Helsinki declaration and its later amendments or comparable ethical standards.

\section{Availability of data and materials:}

The datasets used and/or analyzed during the current study are available from the corresponding author on reasonable request.

\section{Competing interest:}

The authors declare that they have no competing interests

\section{Funding:}

This project did not receive any funding from any agencies in the public, commercial, or non-profit sectors.

\section{References}

1. Encyclopædia Britannica, inc. (n.d.). Hepatic Portal System. Encyclopædia Britannica. Retrieved January 10, 2022, from http://www.britannica.com/science/hepatic-portal-system

2. Item F, Konrad D. Visceral fat and metabolic inflammation: the portal theory revisited. Obes Rev. 2012 Dec;13 Suppl 2:30-9. doi: 10.1111/j.1467-789X.2012.01035.x. PMID: 23107257.

3. Internet archive: Wayback Machine. (n.d.). Retrieved January 10, 2022, from http://archive.org/web/web.php

4. Henderson JM, Nagle A, Curtas S, Geisinger M, Barnes D. Surgical shunts and TIPS for variceal decompression in the 1990 s. Surgery. 2000 Oct;128(4):540-7. doi: 10.1067/msy.2000.108209. PMID: 11015086.

5. Mayo Foundation for Medical Education and Research. (2021, February 20). Esophageal varices. Mayo Clinic. Retrieved January 10, 2022, from https://www.mayoclinic.org/diseases-conditions/esophageal-varices/symptoms-causes/syc20351538\#: :text=Esophageal\%20varices\%20are\%20enlarged\%20veins,throat\%20and\%20stomach\%20(esophagus).

6. Kuo, B., \& Urma, D. (2006, May 16). Esophagus - anatomy and development. Nature News. Retrieved January 10, 2022, from https://www.nature.com/gimo/contents/pt1/full/gimo6.html 
7. The stomach. TeachMeAnatomy. (2018, August 14). Retrieved January 10, 2022, from http://web.archive.org/web/20181006082418/http://teachmeanatomy.info:80/abdomen/gi-tract/stomach/

8. Shaheen AA, Nguyen HH, Congly SE, Kaplan GG, Swain MG. Nationwide estimates and risk factors of hospital readmission in patients with cirrhosis in the United States. Liver Int. 2019 May;39(5):878-884. [PubMed]

9. Yoon H, Shin HJ, Kim MJ, Han SJ, Koh H, Kim S, Lee MJ. Predicting gastroesophageal varices through spleen magnetic resonance elastography in pediatric liver fibrosis. World J Gastroenterol. 2019 Jan 21;25(3):367-377. [PMC free article] [PubMed]

10. Nery F, Correia S, Macedo C, Gandara J, Lopes V, Valadares D, Ferreira S, Oliveira J, Gomes MT, Lucas R, Rautou PE, Miranda HP, Valla D. Nonselective beta-blockers and the risk of portal vein thrombosis in patients with cirrhosis: results of a prospective longitudinal study. Aliment Pharmacol Ther. 2019 Mar;49(5):582-588. [PubMed]

11. Nigatu A, Yap JE, Lee Chuy K, Go B, Doukky R. Bleeding Risk of Transesophageal Echocardiography in Patients With Esophageal Varices. J Am Soc Echocardiogr. 2019 May;32(5):674-676.e2. [PubMed]

12. Stamm ER, Meier JM, Pokharel SS, Clark T, Glueck DH, Lind KE, Roberts KM. Normal main portal vein diameter measured on CT is larger than the widely referenced upper limit of 13 mm. Abdom Radiol (NY). 2016 Oct;41(10):1931-6. doi: 10.1007/s00261-0160785-9. PMID: 27251734.

13. Tidy, D. C. (2014, July 15). Portal hypertension. learn about portal hypertension. Patient.info. Retrieved January 10, 2022, from https://patient.info/doctor/portal-hypertension-pro

14. Dhiman RK, Chawla Y, Vasishta RK, Kakkar N, Dilawari JB, Trehan MS, Puri P, Mitra SK, Suri S. Non-cirrhotic portal fibrosis (idiopathic portal hypertension): experience with 151 patients and a review of the literature. J Gastroenterol Hepatol. 2002 Jan;17(1):6-16. doi: 10.1046/j.1440-1746.2002.02596.x. PMID: 11895549.

15. Mayo Foundation for Medical Education and Research. (2021, February 20). Esophageal varices. Mayo Clinic. Retrieved January 10, 2022, from https://www.mayoclinic.org/diseases-conditions/esophageal-varices/symptoms-causes/syc-

20351538\#: :text=Esophageal\%20varices\%20develop\%20when\%20normal,\%2C\%20causing\%20life\%2Dthreatening\%20bleeding.

16. Bosch J, Pizcueta P, Feu F, Fernández M, García-Pagán JC. Pathophysiology of portal hypertension. Gastroenterol Clin North Am. 1992 Mar;21(1):1-14. PMID: 1568769.

17. Yoshida H, Mamada Y, Taniai N, Yoshioka M, Hirakata A, Kawano Y, Mizuguchi Y, Shimizu T, Ueda J, Uchida E. Risk factors for bleeding esophagogastric varices. J Nippon Med Sch. 2013;80(4):252-9. doi: 10.1272/jnms.80.252. PMID: 23995567.

18. Chakinala RC, Kumar A, Barsa JE, Mehta D, Haq KF, Solanki S, Tewari V, Aronow WS. Downhill esophageal varices: a therapeutic dilemma. Ann Transl Med. 2018 Dec;6(23):463. doi: 10.21037/atm.2018.11.13. PMID: 30603651; PMCID: PMC6312812.

19. NHS. (n.d.). NHS choices. Retrieved January 11, 2022, from https://www.nhs.uk/conditions/cirrhosis/symptoms/

20. U.S. National Library of Medicine. (n.d.). Enlarged liver: Medlineplus medical encyclopedia. MedlinePlus. Retrieved January 11, 2022, from https://medlineplus.gov/ency/article/003275.htm

21. Neetu Radhakrishnan, M. D. (2021, November 5). Splenomegaly. Practice Essentials, Etiology, Epidemiology. Retrieved January 11, 2022, from https://emedicine.medscape.com/article/206208-overview

22. Moore, K. (2003). The management of ascites in cirrhosis: Report on the Consensus Conference of the International Ascites Club. Hepatology, 38(1), 258-266. https://doi.org/10.1053/jhep.2003.50315

23. Longo, D. L. (2012). Harrison's Principles of Internal Medicine Vol. 1 and 2. McGraw-Hill.

24. McPhee, S. J., Papadakis, M. A., \& Rabow, M. W. (2014). 2014 Current medical diagnosis \& treatment. McGraw-Hill Medical.

25. Mayo Foundation for Medical Education and Research. (2021, February 20). Esophageal varices. Mayo Clinic. Retrieved January 11, 2022, from https://www.mayoclinic.org/diseases-conditions/esophageal-varices/diagnosis-treatment/drc-20351544

26. Wang XK, Wang P, Zhang Y, Qi SL, Chi K, Wang GC. A study on spleen transient elastography in predicting the degree of esophageal varices and bleeding. Medicine (Baltimore). 2019 Mar;98(9):e14615. doi: 10.1097/MD.0000000000014615. PMID: 30817578; PMCID: PMC6831430.

27. Zhao W, Liu C, Zhang L, Chong J, Yu N. Application of shear wave elastography as a diagnostic method for esophageal varices. Ann Palliat Med. 2021 Feb;10(2):1342-1350. doi: 10.21037/apm-20-306. Epub 2020 Oct 16. PMID: 33081479.

28. Laine L. Interventions for Primary Prevention of Esophageal Variceal Bleeding. Hepatology. 2019 Apr;69(4):1382-1384. [PubMed]

29. Monreal-Robles R, Cortez-Hernández CA, González-González JA, Abraldes JG, Bosques-Padilla FJ, Silva-Ramos HN, García-Flores JA, Maldonado-Garza HJ. Acute Variceal Bleeding: Does Octreotide Improve Outcomes in Patients with Different Functional 
Hepatic Reserve? Ann Hepatol. 2018 January-February;17(1):125-133. doi: 10.5604/01.3001.0010.7544. PMID: 29311398.

30. Jaspersen D, Schwacha H, Sauer B, Wzatek J, Schorr W, Graf zu Dohna P, Hammar CH. Komplikationen der endoskopischen Sklerotherapie von Osophagusvarizen [Complications of endoscopic sclerotherapy of esophageal varices]. Leber Magen Darm. 1995 Jul;25(4):171-4. German. PMID: 7564871.

31. Pfisterer N, Riedl F, Pachofszky T, Gschwantler M, König K, Schuster B, Mandorfer M, Gessl I, Illiasch C, Fuchs EM, Unger L, Dolak W, Maieron A, Kramer L, Madl C, Trauner M, Reiberger T. Outcomes after placement of a SX-ELLA oesophageal stent for refractory variceal bleeding-A national multicentre study. Liver Int. 2019 Feb;39(2):290-298. [PMC free article] [PubMed]

32. Aggeletopoulou I, Konstantakis C, Manolakopoulos S, Triantos C. Role of band ligation for secondary prophylaxis of variceal bleeding. World J Gastroenterol. 2018 Jul 14;24(26):2902-2914. [PMC free article] [PubMed]

33. Reiberger T, Bucsics T, Paternostro R, Pfisterer N, Riedl F, Mandorfer M. Small Esophageal Varices in Patients with CirrhosisShould We Treat Them? Curr Hepatol Rep. 2018;17(4):301-315. [PMC free article] [PubMed]

34. Kuo SZ, Lizaola B, Hayssen H, Lai JC. Beta-blockers and physical frailty in patients with end-stage liver disease. World J Gastroenterol. 2018 Sep 7;24(33):3770-3775. doi: 10.3748/wjg.v24.i33.3770. PMID: 30197482; PMCID: PMC6127661.

35. Triantos C, Kalafateli M. Endoscopic treatment of esophageal varices in patients with liver cirrhosis. World J Gastroenterol. 2014 Sep 28;20(36):13015-26. doi: 10.3748/wjg.v20.i36.13015. PMID: 25278695; PMCID: PMC4177480.

36. Wu X, Xuan W, Song L. Transjugular intrahepatic portosystemic stent shunt placement and embolization for hemorrhage associated with rupture of anorectal varices. J Int Med Res. 2018 Apr;46(4):1666-1671. doi: 10.1177/0300060517730720. Epub 2018 Jan 17. PMID: 29338471; PMCID: PMC6091825

37. Mishra, P. K., Patil, N. S., Saluja, S., Narang, P., Solanki, N., \& Varshney, V. (2016). High patency of proximal splenorenal shunt: A myth or reality ? - a prospective cohort study. International Journal of Surgery, 27, 82-87.

https://doi.org/10.1016/j.ijsu.2015.12.071

38. COHN R. Surgical treatment of bleeding esophageal varices. Calif Med. 1955 Nov;83(5):348-52. PMID: 13260929; PMCID: PMC1532581.

39. Esophageal varices; causes, symptoms, diagnosis \& treatment. Cleveland Clinic. (n.d.). Retrieved January 11, 2022, from https://my.clevelandclinic.org/health/diseases/15429-esophageal-varices\#: :text=Outlook\%20\%2F\%20Prognosis,What\%20is\%20the\&text=Bleeding\%20esophageal\%20varices\%20is\%20life,at\%20risk\%20for\%20bleeding\%20again.

40. Mayo Foundation for Medical Education and Research. (2021, February 20). Esophageal varices. Mayo Clinic. Retrieved January 11, 2022, from https://www.mayoclinic.org/diseases-conditions/esophageal-varices/symptoms-causes/syc-20351538

41. Rahimi RS, Rockey DC. Complications of cirrhosis. Curr Opin Gastroenterol. 2012 May;28(3):223-9. doi: 10.1097/MOG.0b013e328351d003. PMID: 22343347.

42. Yoshida, Hiroshi et al. "Risk factors for bleeding esophagogastric varices." Journal of Nippon Medical School = Nippon Ika Daigaku zasshi vol. 80,4 (2013): 252-9. doi:10.1272/jnms.80.252

43. Li, Bei et al. "Nonalcoholic Fatty Liver Disease Cirrhosis: A Review of Its Epidemiology, Risk Factors, Clinical Presentation, Diagnosis, Management, and Prognosis." Canadian journal of gastroenterology \& hepatology vol. 2018 2784537. 2 Jul. 2018, doi:10.1155/2018/2784537

44. Triantos, Christos, and Maria Kalafateli. "Endoscopic treatment of esophageal varices in patients with liver cirrhosis." World journal of gastroenterology vol. 20,36 (2014): 13015-26. doi:10.3748/wjg.v20.i36.13015

45. Cao, Zhu-Jun et al. "Bacterial infection triggers and complicates acute-on-chronic liver failure in patients with hepatitis B virusdecompensated cirrhosis: A retrospective cohort study." World journal of gastroenterology vol. 26,6 (2020): 645-656. doi:10.3748/wjg.v26.i6.645

46. Nayagam, Shevanthi et al. "Requirements for global elimination of hepatitis B: a modelling study." The Lancet. Infectious diseases vol. 16,12 (2016): 1399-1408. doi:10.1016/S1473-3099(16)30204-3

47. Hong, Wan-Dong et al. "Predictors of esophageal varices in patients with HBV-related cirrhosis: a retrospective study." BMC gastroenterology vol. 9 11. 5 Feb. 2009, doi:10.1186/1471-230X-9-11 